\title{
Quality Assurance in SPECT/CT for Radionuclide Therapy with Lutetium-177
}

\section{Olivia Adu-Poku ${ }^{1,2 *}$, Eric Kotei Addison 1,2, Bright Kwakye-Awuah1, Stephen Inkoom ${ }^{3,4}$, Francis Hasford 3 , Cletus Ahadzie5, Joseph Adom 1,2, Isaac Kojo Wilson ${ }^{3}$}

\author{
${ }^{1}$ Department of Physics, Kwame Nkrumah University of Science and Technology, Kumasi, Ghana \\ ${ }^{2}$ Komfo Anokye Teaching Hospital, Oncology Directorate, Department of Medical Physics, Kumasi, Ghana \\ ${ }^{3}$ Department of Medical Physics, Graduate School of Nuclear and Allied Sciences, University of Ghana, Accra, Ghana \\ ${ }^{4}$ Radiation Protection Institute, Ghana Atomic Energy Commission, Accra, Ghana \\ ${ }^{5}$ Nuclear Regulatory Authority, Ghana Atomic Energy Commission, Accra, Ghana \\ Email: *missolive70@ymail.com
}

How to cite this paper: Adu-Poku, O., Addison, E.K., Kwakye-Awuah, B., Inkoom, S., Hasford, F., Ahadzie, C., Adom, J. and Wilson, I.K. (2022) Quality Assurance in SPECT/CT for Radionuclide Therapy with Lutetium-177. International Journal of Medical Physics, Clinical Engineering and Radiation Oncology, 11, 60-76.

https://doi.org/10.4236/ijmpcero.2022.111006

Received: December 16, 2021

Accepted: February 21, 2022

Published: February 24, 2022

Copyright (c) 2022 by author(s) and Scientific Research Publishing Inc. This work is licensed under the Creative Commons Attribution International License (CC BY 4.0).

http://creativecommons.org/licenses/by/4.0/ (c) (i) Open Access

\begin{abstract}
This study was done to quality assure the Hawkeye SPECT/CT at the St. Olav's hospital and create a clinical method for doing individual dosimetry with ${ }^{177} \mathrm{Lu}$-octreotate in targeted radionuclide therapy for neuroendocrine tumors. Various quality control parameters were performed on Infinia Hawkeye SPECT/CT. A calibration dose of $160 \% \pm 2 \% \mathrm{MBq}$ was ordered and first calibrated for all the dose calibrators. The uniformity test was obtained using a $40 \mathrm{MBq}$ Tc-99m point source positioned $2.5 \mathrm{~m}$ away from the two detectors. A $200 \mathrm{MBq}$ Tc-99m was diluted in $70 \mathrm{ml}$ of water, dispersed in six syringes for the registration test. A Lu-177 point source was placed in front of the detectors, one at a time, to check the energy peaks. The Jaczczak phantom with a hollow sphere set (volumes: $0.5,1,2,4,8$, and 16) $\mathrm{ml}$ with an additional 60 $\mathrm{ml}$ sphere was used for the 3D sensitivity and recovery with Lu-177. Total activity of $945.3 \mathrm{MBq}$ was added to $160 \mathrm{ml}$ of water yielding an activity concentration of $5.908 \mathrm{MBq} / \mathrm{ml}$ in the spheres. The phantom was then scanned at various time intervals. A cylindrical phantom with a volume of $6283 \mathrm{ml}$ was also used to obtain the cross-calibration measurement (cps/MBq). Total activity of 995.6 MBq was added and the phantom was scanned at days 0, 6, 13 and 23 . The dose calibration factor was changed from 762 to 760 to achieve correct doses. The 2D mean sensitivity factor was $5.56 \mathrm{cps} / \mathrm{MBq}$. Uniformities for both detectors were approved after iteration calibration of the PM tubes. The X-ray to SPECT registration was found to be accurate and within specifications. The energy peak test revealed off-centered $208 \mathrm{keV}$ energy peaks for the two detectors. Quality assurance of imaging devices using radiation is essential for radiation protection and ensures a high-quality image.
\end{abstract}




\section{Keywords}

Lutetium-177, Activity Concentration, Dose Calibrator, SPECT/CT

\section{Introduction}

Medical imaging provides tremendous and undeniable benefit in modern health care. It is used for disease detection, classification, prognostic staging, treatment planning and to validate therapeutic response [1]. Single-photon emission computed tomography with X-ray computed tomography (SPECT/CT) is a nuclear medicine tomographic imaging technique which enhances accuracy in diagnostic for specific medical indications from likely attenuation and/or the scatter correction of the SPECT functional images and the availability of helpful anatomic information [2]. From literature data, SPECT-CT compared to only SPECT gives more radiation to the patient which may not cause deterministic effects. The effective dose for SPECT is dependent on the administered activity and the age of the patient. The average radiopharmaceutical effective dose changes from tens to thousands of $\mathrm{mSv}$ for some nuclear medicine examinations [3]. Nonetheless, the introduction of CT in nuclear diagnostic procedures causes a momentous increase in the patient dose. Generally, effective doses (E) for CT examinations can be higher than most other diagnostic imaging modalities [4]. In the hybrid imaging modalities operation in nuclear medicine, aside from the internal radiation exposure emanating from the administration of radiopharmaceuticals, the external radiation exposure (X-ray) resulting from the CT device has to be considered in light of International Commission on Radiological Protection (ICRP) recommendations [5] [6]. These recommendations take account of the justification of practices (the use of radiation produces sufficient benefit to offset any risks caused by the use of radiation), optimization (the incurred exposure by the use of radiation should be kept as low as reasonably achievable), and dose limitation. The internal radiation exposure doses of each organ after radiopharmaceutical administration are calculated by the MIRD method [7].

Today, due to improvements in conventional treatment modalities, namely, surgery, external radiotherapy, chemotherapy, and biotherapy, an increasing number of cancer patients are successfully treated. Nonetheless, therapy fails in about one-third of patients mainly due to metastatic spread, while new therapy approaches under development, such as Targeted Radionuclide Therapy (TRT), usually focus on distant metastasis treatment. Currently, Re-186, Y-90, I-131 and Lu-177 are the most often used radionuclides. Lu-177 has risen in prominence as a result of its increasing application in Peptide Receptor Radionuclide Therapy (PRRT). Numerous groups have developed new 3D methods for the better and more patientspecific quantification [8] [9] [10]. Several radionuclides were proposed and evaluated in neuroendocrine tumor radionuclide therapy. An early attempt was to use ${ }^{111}$ In [11], with positive results. However, since the cross-fire dose to healthy 
tissues is high, due to its significant gamma emission, ${ }^{111} \mathrm{In}$ is not a suitable radionuclide treatment. Later, ${ }^{90} \mathrm{Y}$, emitting pure high-energy beta particles, and recently ${ }^{177} \mathrm{Lu}$, emitting low-energy beta particles with a low gamma radiation emission were applied [12]-[17]. It was also proposed that ${ }^{114 \mathrm{~m}} \mathrm{In}$, which emits both quantifiable gamma radiation and a combination of high and low-energy beta particles and electrons [18], and ${ }^{64} \mathrm{Cu}$, which emits positrons and low-energy beta particles [19] [20] [21], may be used. From their papers, the following quotes were given:

"Only ${ }^{177} \mathrm{Lu}$ is capable of producing a sufficiently high cell kill in tumors of all sizes, although its effect in larger tumors is reduced".

"Remarkably, ${ }^{177} \mathrm{Lu}$ is able to cure tumors of small- and medium-size over the entire range of clonogen densities".

Their results are significant and indicate that the clinical outcome to these patients could be significantly improved if ${ }^{177} \mathrm{Lu}$ is included in the patient care. This work aimed to quality assure the Hawkeye SPECT/CT and develop a feasible clinical way of performing individual dosimetry using $177 \mathrm{Lu}$-octreotate in targeted neuroendocrine tumor radionuclide therapy.

\section{Materials and Method}

\subsection{Calibration of Dose Calibrators to Lu-177}

The Institute of Fire Engineers (IFE) was contacted and a calibration dosage of $160 \% \pm 2 \% \mathrm{MBq}$ Lu-177 with batch number 20-02201 was ordered. The IBC NM and IBC Lite dosage calibrators were employed in this study. The vial was from Tripoli. Lu-177 was used for the purpose of calibration of dose calibrators. To simulate a typical patient dose, the volume was diluted to $20 \mathrm{ml}$ from $10 \mathrm{ml}$. The vial's source was placed in the source holder before being placed in the dose calibrator. It was calibrated to 2020-02-03, 12.00 UTC time. The dose calibration factors initially on the IBC NM and IBC Lite were 762 and 751 respectively. The activity data from the dosage calibrator were compared to the activity measurement provided by IFE after allowing for decay. Adjustments were made to the dose calibrator measurements to bring them into line with the activity data provided by IFE. The revised settings were utilized as a baseline for Lu-177 activity assessments. At the moment of measurement, the calibration factor was changed to get the expected activity decay-corrected value. Figure 1(a) and Figure 1(b) show the dose calibrator and the activity display in MBq respectively.

\subsection{Uniformity Test with Tc-99m}

This test was done using a $40 \mathrm{MBq}$ Tc-99m point source positioned $2.5 \mathrm{~m}$ away from the two detectors as shown in Figure 2. For each detector, static acquisitions of 60,000 counts were made separately. Iterative calibrations were executed by entering Calibration -> NM maps creation -> Uniformity Map creation with the background confirmed to be zero. The uniformity test data was collected. 
For detector 2, the technique was repeated.

\subsection{X-Ray to SPECT Registration Text}

This test was carried out with the use of a specific alignment phantom with six holes made of low-attenuation material. About $200 \mathrm{MBq}$ Tc-99m was diluted in $70 \mathrm{ml}$ of water and dispersed in six syringes. The distances between the CT and SPECT were measured using these sources as landmarks. The syringes were inserted into the GE X-ray to NM registration phantom as shown in Figure 3(a)

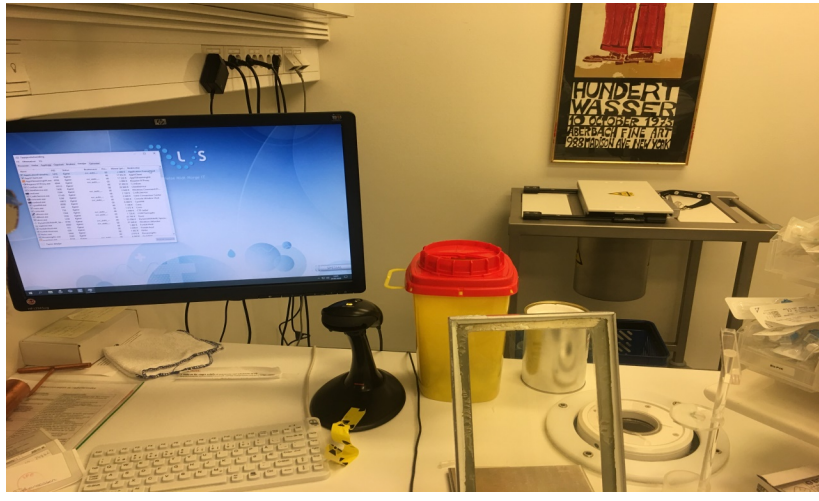

(a)

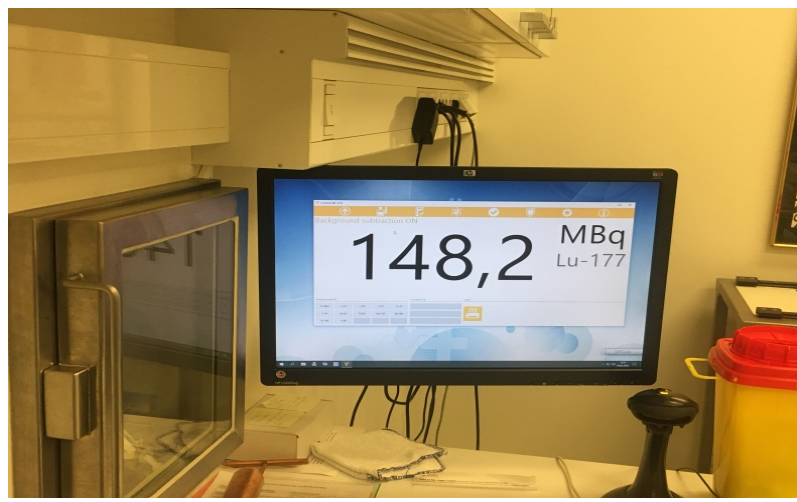

(b)

Figure 1. (a) The dose calibrator; (b) Display of activity value in MBq on dose calibrator screen.

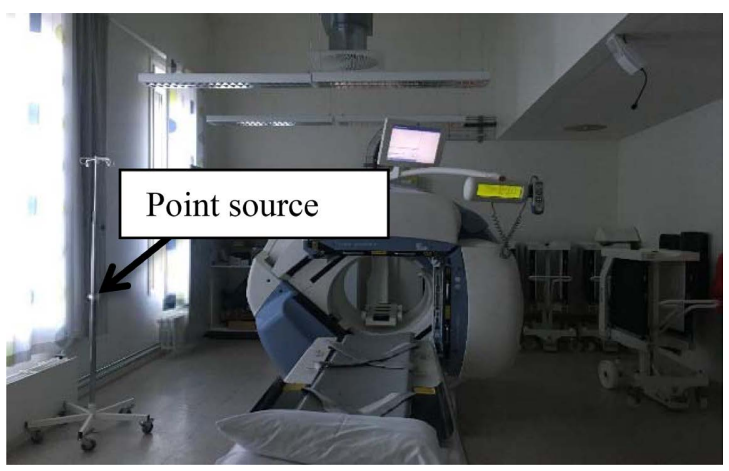

Figure 2. Uniformity test.

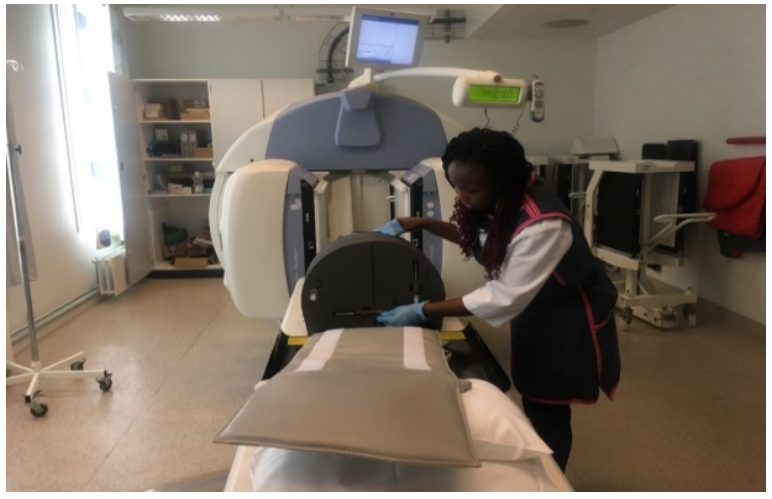

(a)

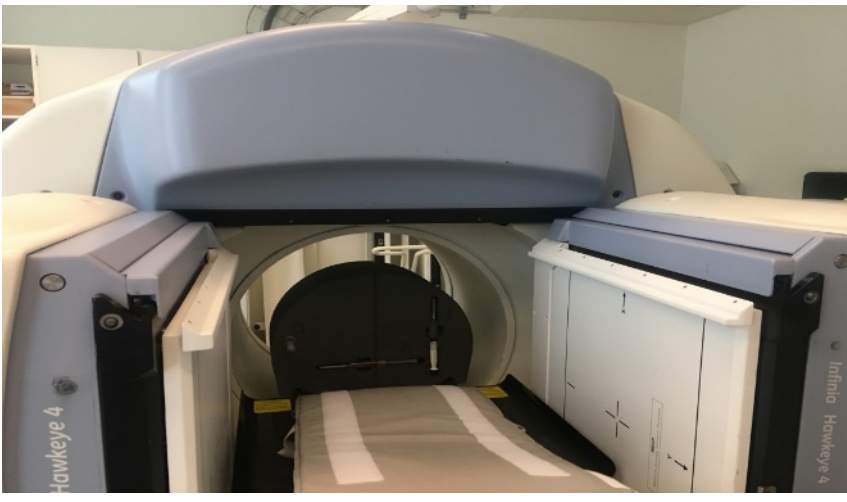

(b)

Figure 3. (a) Placing hot syringes in phantom holes; (b) Phantom placed on couch for SPECT/CT acquisition. 
and Figure 3(b) and the registration test was done. To mimic a patient, a 10 - 15 $\mathrm{kg}$ weight was placed in the end of the pallet and the Hawkeye Registration QC program applied in the evaluation.

\subsection{Energy Peak Calibration with Lu-177}

To check the energy peaks of the isotope, a $\mathrm{Lu}-177$ point-source was placed before each detector one at a time (113 keV and $208 \mathrm{keV})$. Both detectors showed off-centered $208 \mathrm{keV}$-energy peaks during the test (between 200 and $205.5 \mathrm{keV}$ ). A calibration of the energy peak was carried out. To ensure that the calibration was correct, a static acquisition was done. On the Xeleris workstation, an energy peak calibration was performed, (first peak $113 \mathrm{keV}$, then peak 208 for each detector) A static acquisition (Utilities -> NM and X-ray acq -> Static acq and PHA, corrections: Energy session Lu-177 (113 208 178), intrinsic -> Backup Energy correction maps) was used to check the calibration.

\subsection{D Sensitivity with Lu-177}

In an $8.5 \mathrm{~cm}$ diameter petri dish, a thin layer of water was poured. A total activity of $82 \mathrm{MBq}$ was added to the water to obtain the $2 \mathrm{D}$ sensitivity. As illustrated in Figure 4, the contents of the petri dish were put at a distance of $10 \mathrm{~cm}$ from each detector. The energy peak window and scatter window were set to $208 \% \pm 10 \%$ $\mathrm{KeV}$ and $178 \% \pm 5 \% \mathrm{KeV}$, respectively, using a 180 -second static acquisition protocol. To collect the resulting count from each detector, 1335-pixel-wide regions of interests (ROIs) were drawn on each image acquired at the Xeleris workstation. The counts per second per $\mathrm{MBq}$ (cps/MBq) were used to calculate the sensitivity.

\subsection{Cross Calibration Measurement}

For a precise 3D sensitivity activity estimation and quantification, a cylindrical phantom with a capacity of $6283 \mathrm{ml}$ was used. The phantom was filled with water and lutetium-177 with an activity of $995.6 \mathrm{MBq}$ was dispensed, producing an initial activity concentration of $158.5 \mathrm{kBq} / \mathrm{ml}$. The phantom was then vigorously shaken to even out the activity dispersion. The phantom was positioned on the

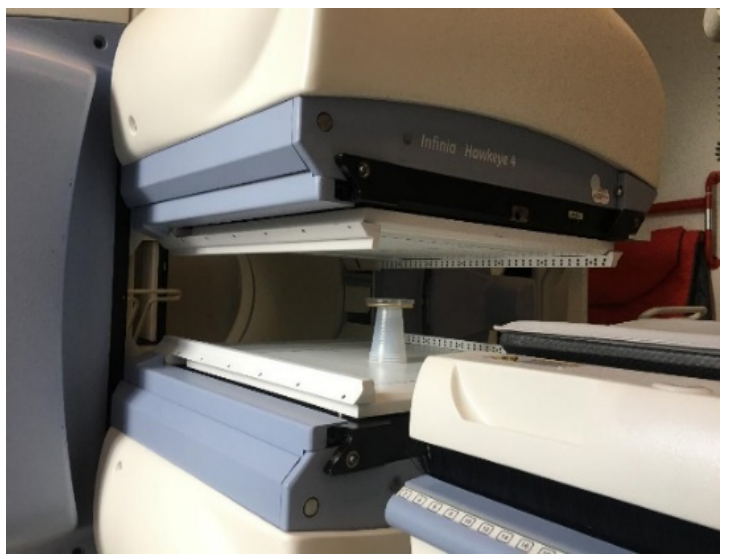

Figure 4. Lutetium-177 placed $10 \mathrm{~cm}$ away from each of the detectors. 
treatment couch as illustrated in Figure 5 below and scanned at days $0,6,13$, and 20. The reconstructions were carried out in the same way as the patient investigations, according to the clinical protocol.

\subsection{The 3D Sensitivity and Recovery with Lu-177}

The Jaszczak phantom was used, along with hollow sphere sets with capacities of $0.5,1,2,4,8,16 \mathrm{ml}$, and a $60 \mathrm{~mm}$ sphere with a volume of roughly $100 \mathrm{ml}$. The phantom was first filled with water and there was no background activity, as illustrated in Figure 6(a). A total of $945.3 \mathrm{MBq}$ of activity was added to a $160 \mathrm{ml}$ of water and then dispensed into the spheres, giving an activity concentration of $5.904 \mathrm{MBq} / \mathrm{ml}$ in every sphere. This activity concentration is similar to tumor levels of around $5 \mathrm{MBq} / \mathrm{ml}$ in the first week following Lu-177 treatment. The phantom was placed on the couch as seen in Figure 6(b) and scanned at days $0,7,13$, and 20 . The acquisition of the $3 \mathrm{D}$ sensitivity test was then performed.

\section{Results and Discussion}

\subsection{Calibration of Dose Calibrators to Lu-177}

The dose calibration factor in IBC NM was modified from 762 to 760 to get correct

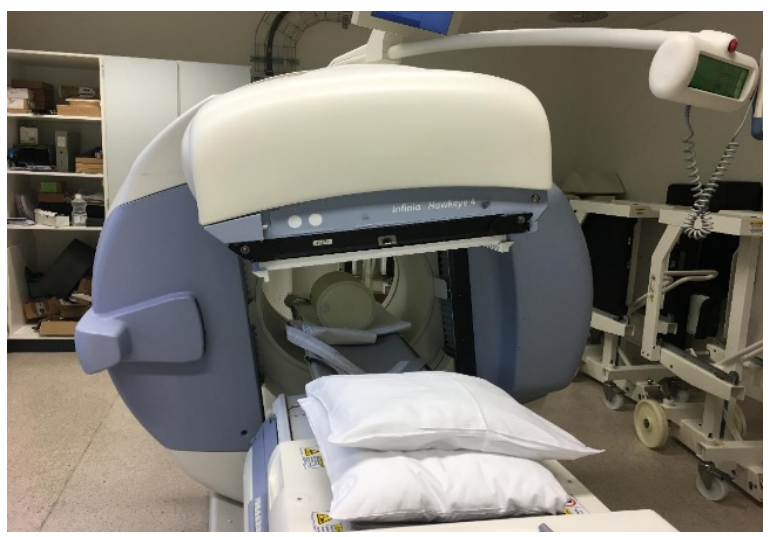

Figure 5. Cross calibration phantom set up on Infinia Hawkeye 4 SPECT/CT system.

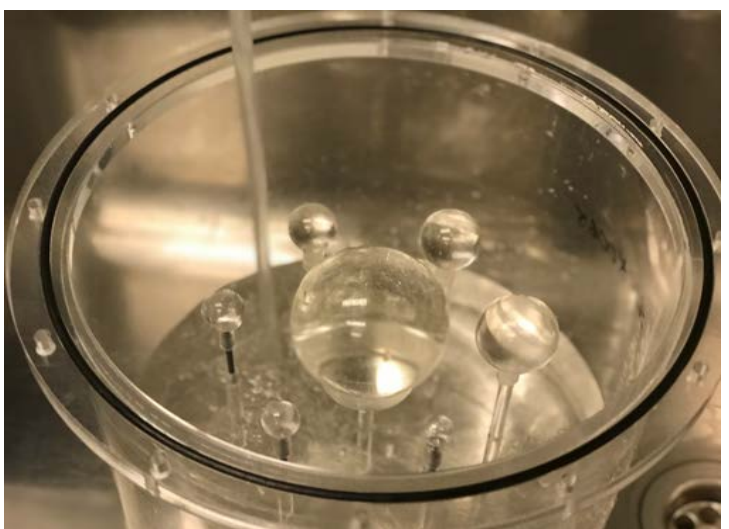

(a)

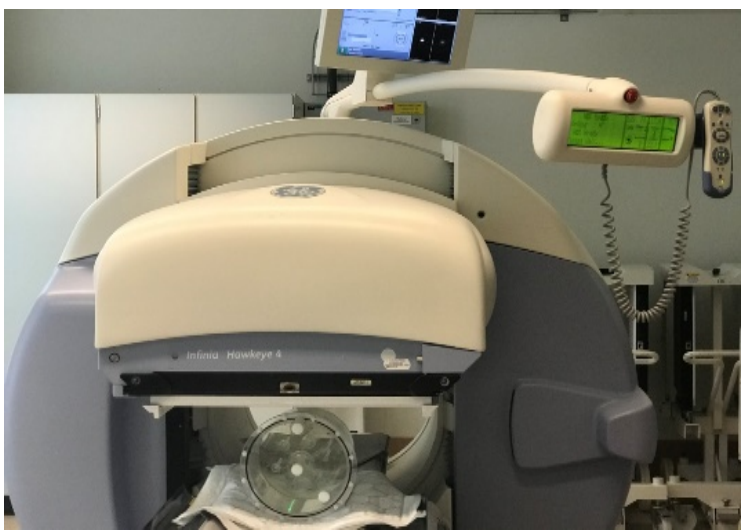

(b)

Figure 6. (a) Filling background with water; (b) 3D sensitivity test acquisition. 
doses for Lu-177. The calibration factor could not be altered with the IBC Lite dosage calibrators since it was not possible to save a new dose calibration factor in the IBC Lite. For Lu-177 measurements, IBC NM dosage calibrators in the injection room and Hotlabs 1 should be used. The IBC NM dosage calibrator at Hotlab 2 and the IBC Lite dose calibrators should not be used until service confirms correct dose calibration parameters. Table 1 gives the measured doses and deviation from calibrated Lu-177-activity for all dose calibrators at the nuclear medicine department of the St Olav's hospital.

\subsection{Uniformity Test with Tc-99m (Intrinsic)}

The uniformity test checks that the detector's response to a source is consistent and uniform within specified limits. The Useful Field of View (UFOV) and the Centred Field of View (CFOV) were the two essential parameters used to evaluate this test. Static acquisitions with 60,000 kcts were acquired separately for the two detectors. As demonstrated in Table 2 and Figure 7, the uniformity for detector 1 was within acceptable limits, however uniformity for detector 2

Table 1. Measured doses and deviation from calibrated Lu-177-activity for all dose calibrators.

\begin{tabular}{ccc}
\hline Dose calibrator & Measured dose (MBq) & Deviation [\%] \\
\hline IBC NM (762) & 159.38 & \\
Injection room & 159.57 & -0.39 \\
Hotlab 1, large bench & 159.58 & -0.27 \\
Hotlab 1, small bench & 162.74 & -0.26 \\
Hotlab 2 & & 1.71 \\
IBC Lite (751) & 165.52 & \\
Injection room & 165.51 & +3.45 \\
Hotlab 1, large bench & 159.73 & +3.45 \\
Hotlab 1, small bench & 163.28 & -0.17 \\
Hotlab 2 & & +2.05 \\
\hline
\end{tabular}

Table 2. Results from uniformity test with Tc-99m prior to iterative calibration of PM-tubes.

\begin{tabular}{cccc}
\hline Measurement & Value Detector 1 & Value Detector 2 & Acceptance \\
\hline CFOV Y-Diff & $1.039318301 \%$ & $1.171576352 \%$ & $\leq 2.1$ \\
UFOV Integral & $2.28865223 \%$ & $4.462838167 \%$ & $\leq 3.6$ \\
UFOV X-Diff & $1.361474989 \%$ & $4.158851242 \%$ & $\leq 2.3$ \\
CFOV Integral & $1.98408687 \%$ & $2.114286743 \%$ & $\leq 3.0$ \\
CFOV X-Diff & $1.361474989 \%$ & $1.337810855 \%$ & $\leq 2.1$ \\
UFOV Y-Diff & $1.104751909 \%$ & $2.37668436 \%$ & $\leq 2.3$ \\
Energy Peak & $140.794 \mathrm{keV}$ & $139.965 \mathrm{keV}$ & $140 \pm 3$ \\
\hline
\end{tabular}


failed. The PM tubes were iteratively calibrated, and a new uniformity test was obtained as a result. As shown in Table 3 and Figure 8, uniformity was accepted after iteration of the PM tubes. Table 4 gives the results for the uniformity test
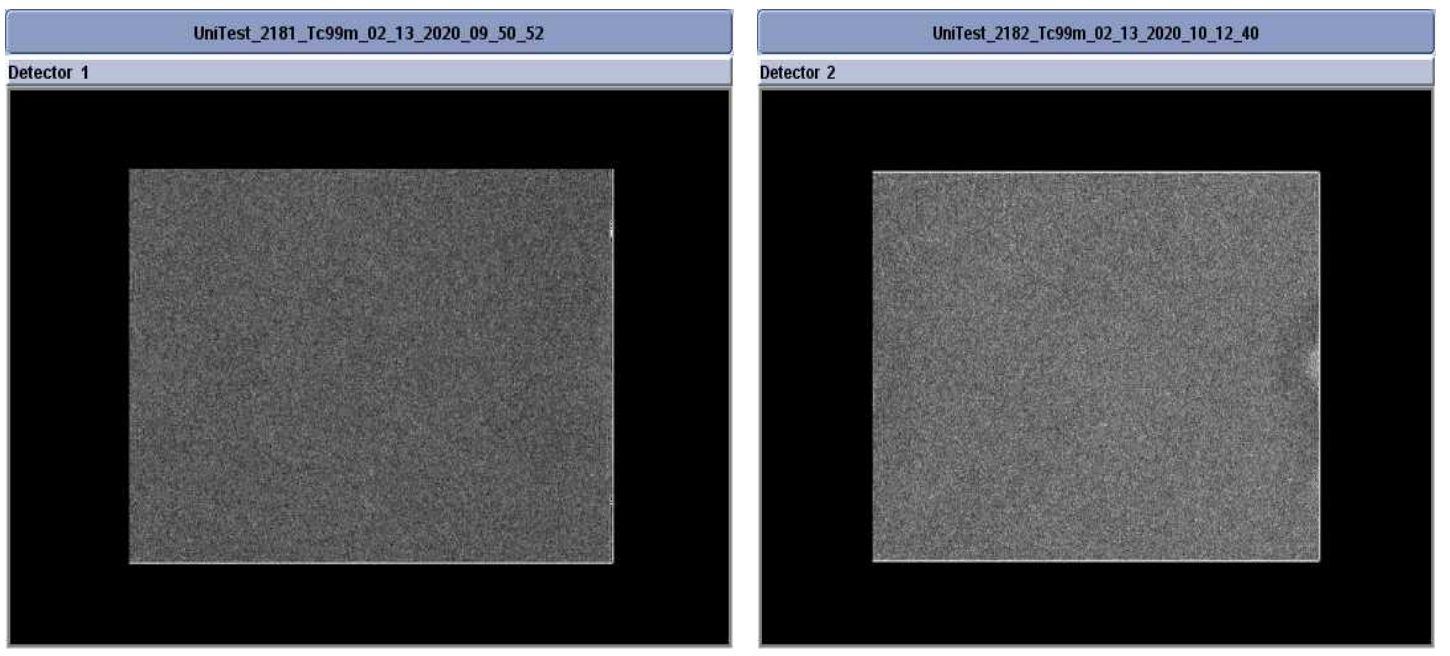

Figure 7. Images from uniformity test with Tc-99m prior to iterative calibration of PM-tubes (512 matrix).

Table 3. Results from uniformity test with Tc-99m after iterative calibration of PM-tubes.

\begin{tabular}{cccc}
\hline Measurement & Value Detector 1 & Value Detector 2 & Acceptance \\
\hline CFOV Y-Diff & $0.7217389425 \%$ & $0.4323223901 \%$ & $\leq 2.1$ \\
UFOV Integral & $1.496589951 \%$ & $1.059591629 \%$ & $\leq 3.6$ \\
UFOV X-Diff & $1.123888105 \%$ & $0.6231776135 \%$ & $\leq 2.3$ \\
CFOV Integral & $1.10905065 \%$ & $0.9435581492 \%$ & $\leq 3.0$ \\
CFOV X-Diff & $0.8739763991 \%$ & $0.5565401909 \%$ & $\leq 2.1$ \\
UFOV Y-Diff & $0.8860338435 \%$ & $0.5439039327 \%$ & $\leq 2.3$ \\
Energy Peak & $139.017 \mathrm{keV}$ & $138.133 \mathrm{keV}$ & $140 \pm 3$
\end{tabular}
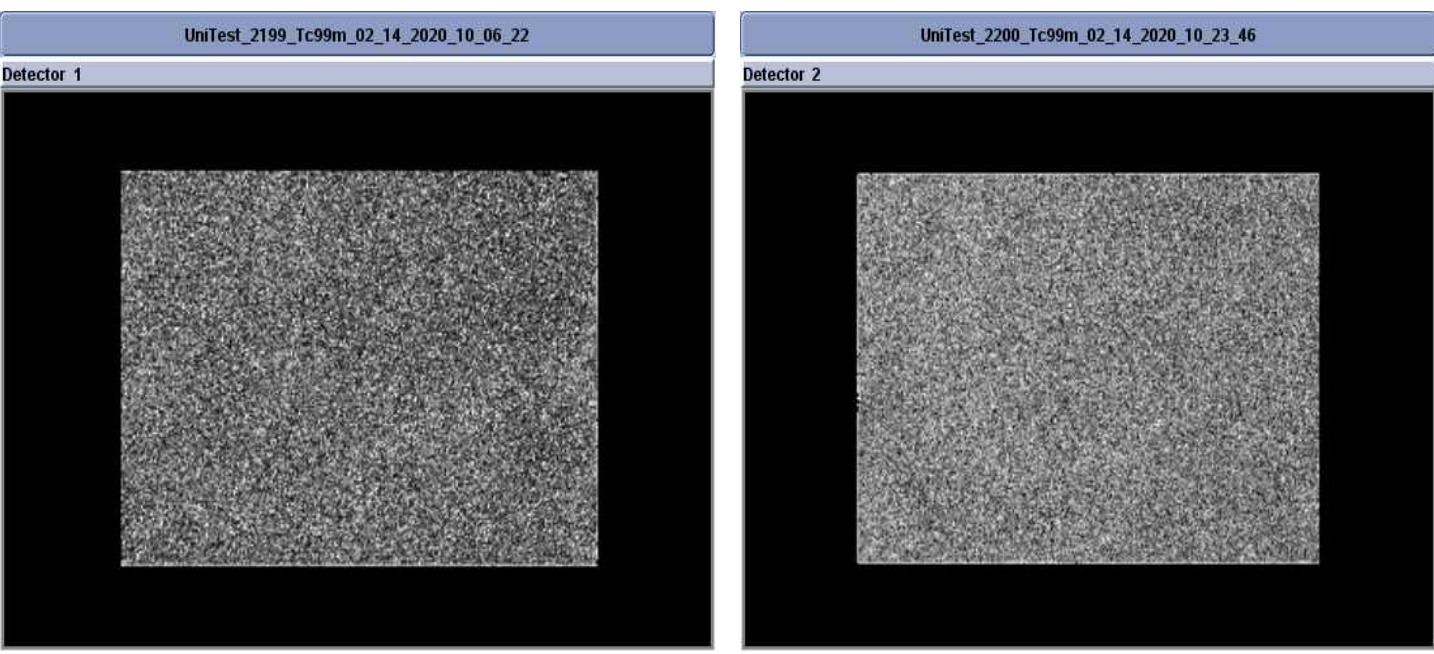

Figure 8. Images from uniformity test with Tc-99m after iterative calibration of PM-tubes. 
for both detectors. The acquisition time was $645.0 \mathrm{sec}$ and $698 \mathrm{sec}$ for detectors 1 and 2 respectively. The Full Width Half Maximum (FWHM) for detector1 was 9.745\% whiles that for detector 2 was 9.560\%. Figure 9(a) and Figure 9(b) illustrate the detector images for detectors 1 and 2 showing their energy peaks respectively.

Table 4. Detector results for uniformity test for both detectors.

\begin{tabular}{ccc}
\hline Name & Detector 1 value & Detector 2 value \\
\hline Acquisition total time & $645.0 \mathrm{sec}$ & $698.0 \mathrm{sec}$ \\
FWHM & $9.745 \%$ & $9.560 \%$ \\
Energy Peak & $140.794 \mathrm{keV}$ & $139.965 \mathrm{keV}$ \\
Total count & $60,000.0 \mathrm{kcts}$ & $59,999.0 \mathrm{kcts}$ \\
Average event rate & $92.9 \mathrm{kc} / \mathrm{s}$ & $86.0 \mathrm{kc} / \mathrm{s}$ \\
\hline
\end{tabular}
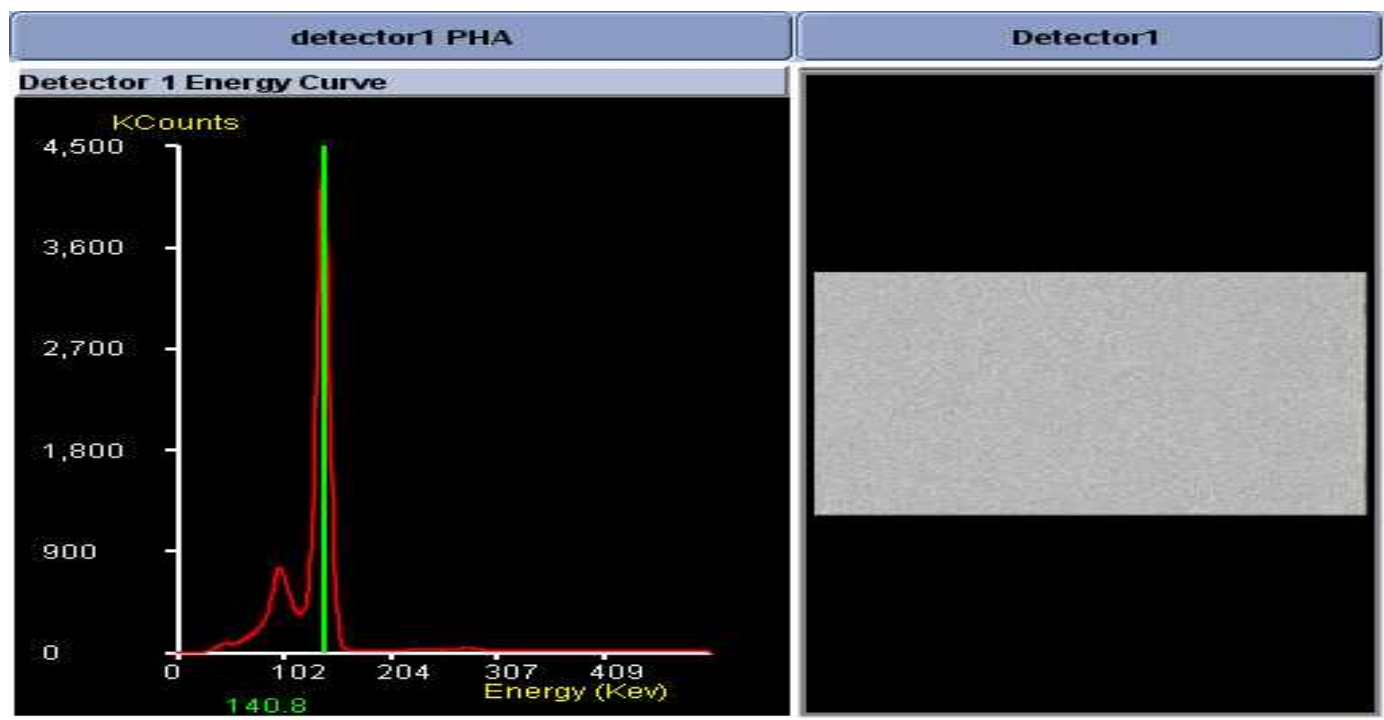

(a)

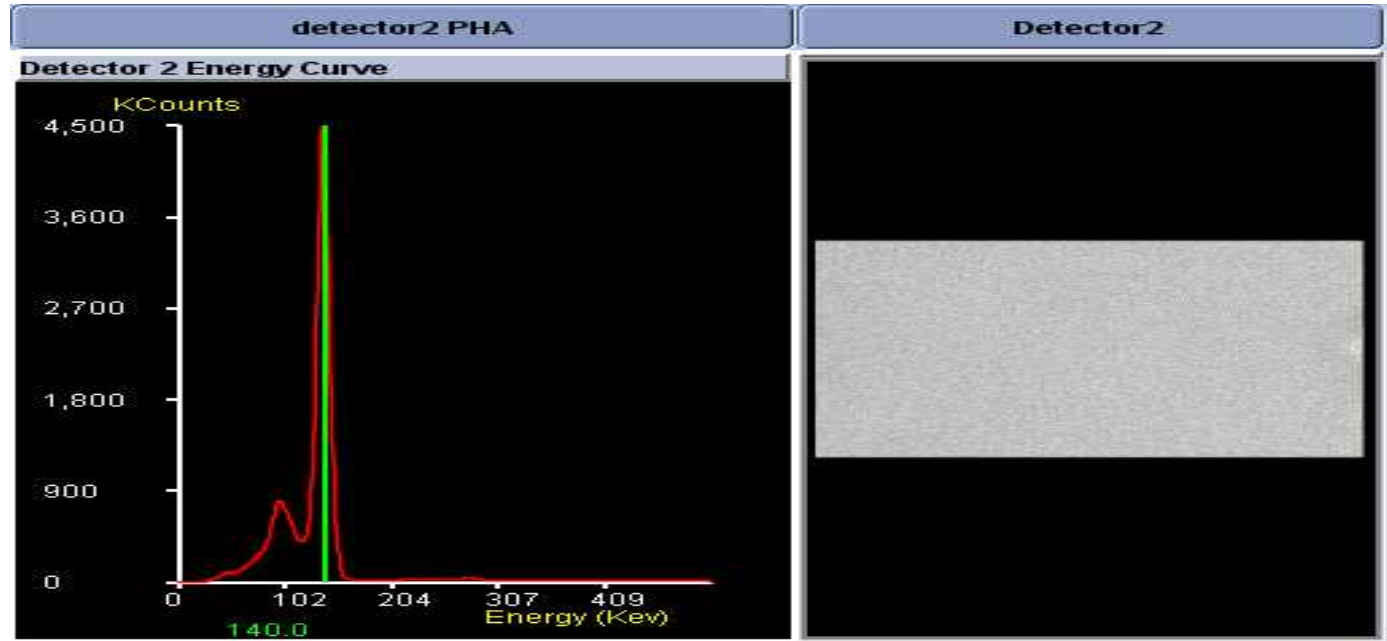

(b)

Figure 9. (a) Detector image for detector 1 their energy peaks; (b) Detector image for detector 2 showing their energy peaks. 


\subsection{X-Ray to SPECT Registration Text}

The registration test (tomo) was acquired with mounted MEGP collimators. Hawkeye Registration QC program on Xeleris workstation was used in the evaluation as shown in Figure 10 below. The registration from X-ray to SPECT registration was precise, accurate and within specifications as seen in Table 5.

\subsection{Energy Peak}

The test revealed that the two detectors had off-centered $208 \mathrm{keV}$ energy peaks (between 200 and $205.5 \mathrm{keV}$ ), so an energy peak calibration was performed. To validate the calibration, a static acquisition was also done (by entering Utilities-> NM and X-ray acq -> Static acq and PHA, corrections: Energy session Lu-177 (113 208 178), intrinsic -> Backup Energy correction maps). The result after calibration of both detectors is demonstrated Table 6 .

\subsection{D Sensitivity}

The sensitivity of an imaging device is the fraction of incident photons that it records. The count for each slice and sleeve was calculated using the decay correction

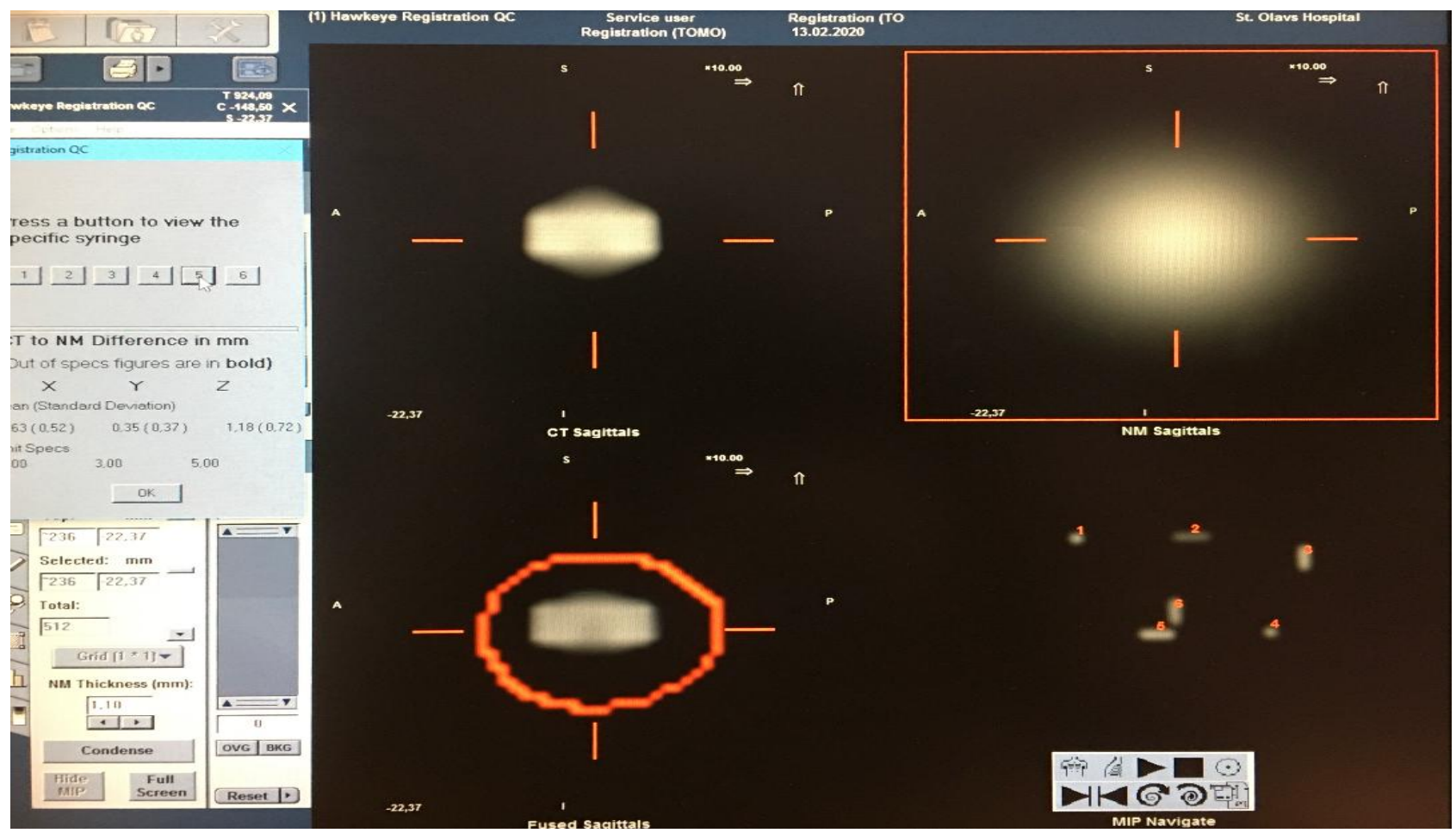

Figure 10. Running Hawkeye registration QC program on Xeleris.

Table 5. CT to NM difference.

\begin{tabular}{cccc}
\hline Measurement & $\mathrm{X}$ & $\mathrm{Y}$ & $\mathrm{Z}$ \\
\hline Mean (standard deviation) & $0.63(0.52)$ & $0.35(0.37)$ & $1.18(0.72)$ \\
Limit specs & 3.00 & 3.00 & 5.00 \\
\hline
\end{tabular}


algorithm and attenuation correction for each sleeve. To obtain the counts from each detector, areas of interest (ROIs) of 1360 pixels were drawn on each image acquired at the Xeleris workstation. The counts per second per $\mathrm{MBq}$ were used to calculate the $2 \mathrm{D}$ sensitivity, and the mean $2 \mathrm{D}$ sensitivity was found to be 5.56 cps/MBq. For a clinical protocol with measurement duration of 340 s/pixel (7 $\mathrm{cm} / \mathrm{min}$ ), this equates to 1889 counts/MBq. The number of counts acquired and the computed $2 \mathrm{D}$ sensitivity for the two detectors are listed in Table 7. Images acquired at the Xeleris workstation to get the resulting count from each detector are shown in Figure 11. Figure 12 also gives the 2D sensitivity factor graph for the two detectors and the mean sensitivity.

\subsection{D Calibration}

To carry out quantitative imaging with whole body scanning technique as well as

Table 6. Energy peaks after calibration of both detectors.

\begin{tabular}{ccc}
\hline Detector & Energy peak $113 \mathrm{keV}$ & Energy peak 208 keV \\
\hline Detector 1 & 113.4 & 209.755 \\
Detector 2 & 112.9 & 208.225 \\
\hline
\end{tabular}

Table 7. Acquired counts and calculated 2D sensitivity for both detectors.

\begin{tabular}{cccc}
\hline Detector & $\begin{array}{c}\text { Counts } \\
\text { (emission) }\end{array}$ & $\begin{array}{c}\text { Counts } \\
\text { (scatter) }\end{array}$ & $\begin{array}{c}\text { 2D sensitivity (emission } \\
\text { only) [cps/MBq] }\end{array}$ \\
\hline Detector 1 & 81,640 & 4560 & 5.531 \\
Detector 2 & 82,325 & 4285 & 5.581 \\
Total (detector 1 \& 2) & 163,965 & 8845 & 11.112 \\
Mean & 81,982 & 4422 & 5.560 \\
\hline
\end{tabular}

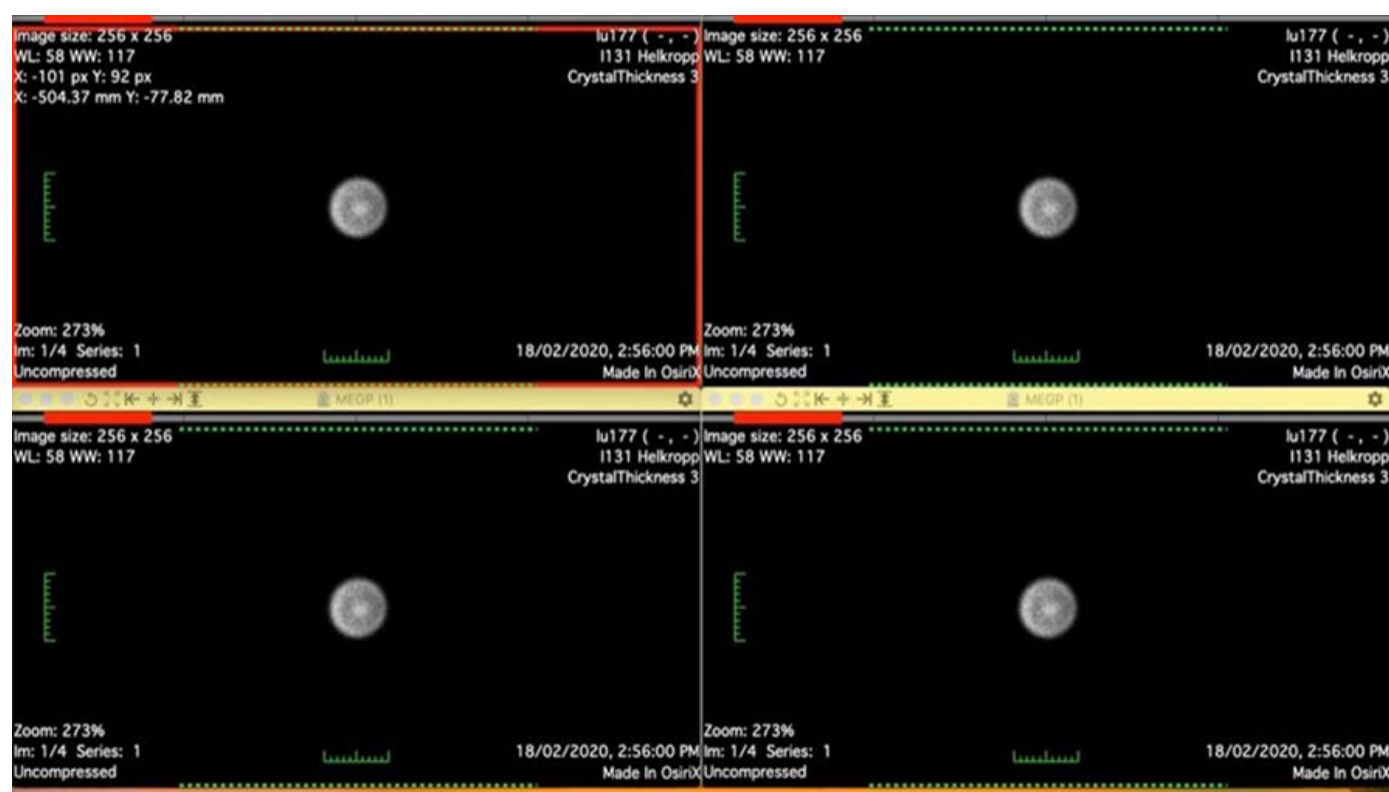

Figure 11. Images acquired at the Xeleris workstation to get the resulting count from each detector. 
SPECT, the camera needed to be calibrated to obtain sensitivity factors converting counts to activity. The scanned phantom was imported onto an OXIRIS image analysis tool for the analysis. The number of counts was obtained to determine the $3 \mathrm{D}$ sensitivity (calibration factor) for accurate activity quantification for each scan date and time. The activity dropped with time whilst the sensitivity increased with time. Figure 13 and Figure 14 show the phantom scans and the

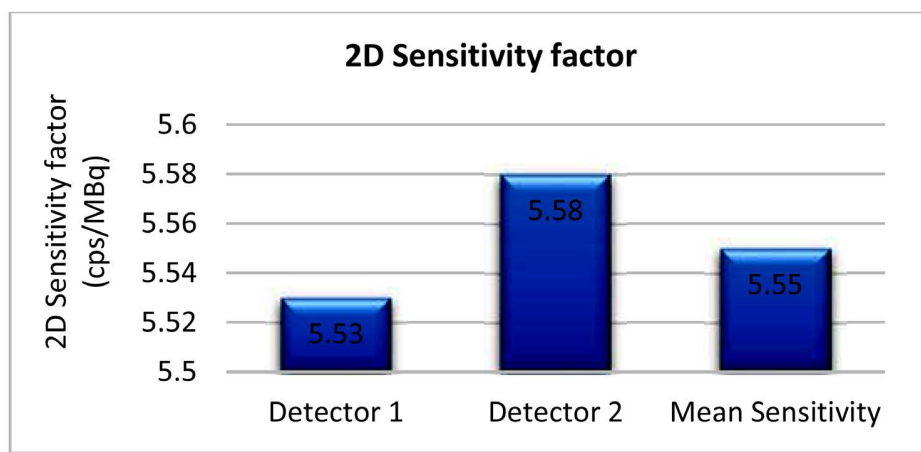

Figure 12. A 2D sensitivity factor graph for the two detectors and the mean sensitivity.

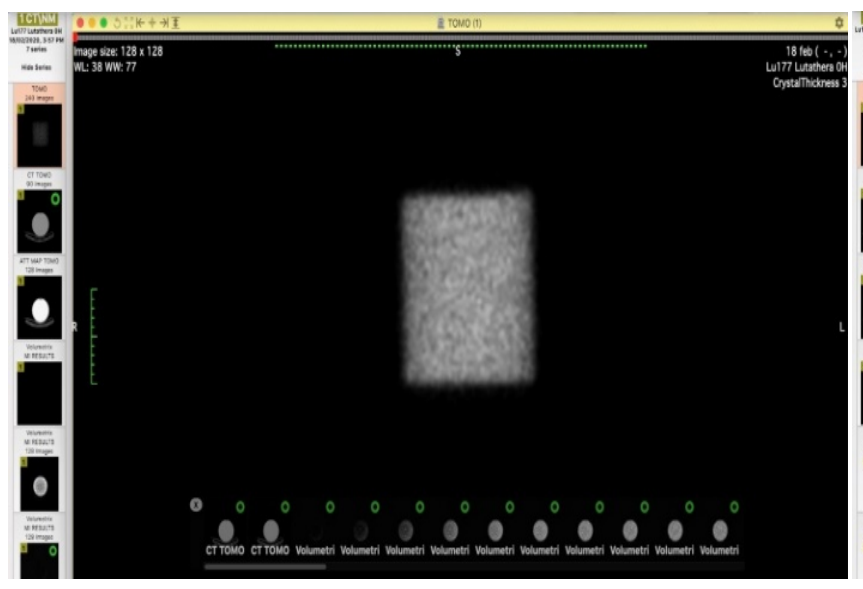

(a)

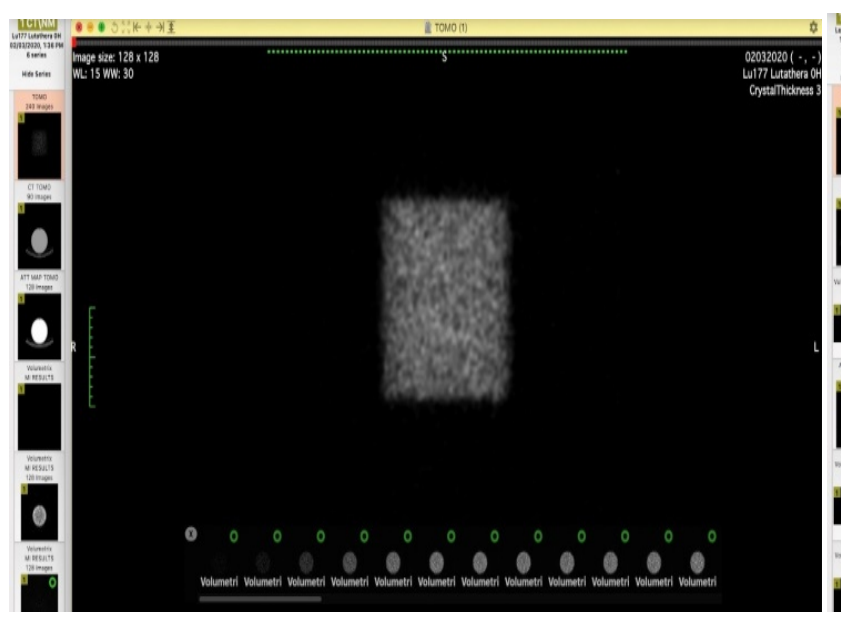

(c)

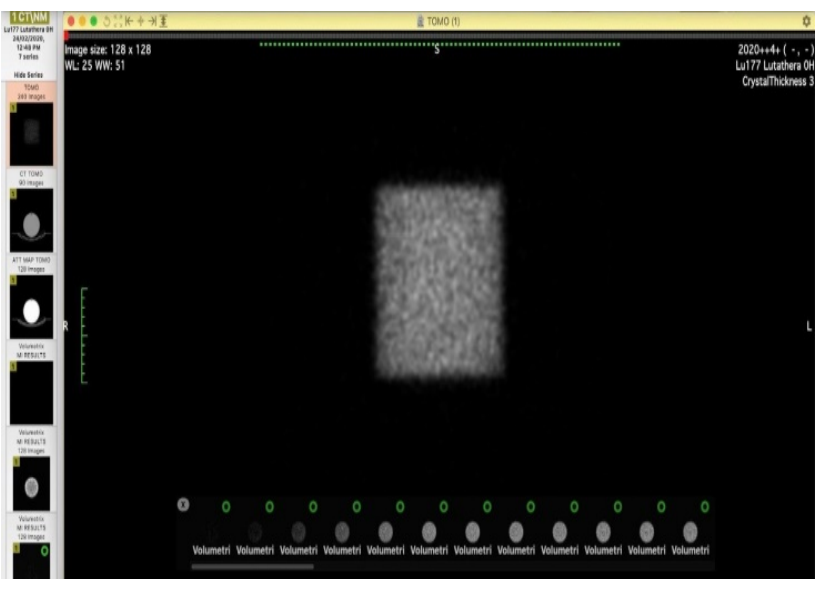

(b)

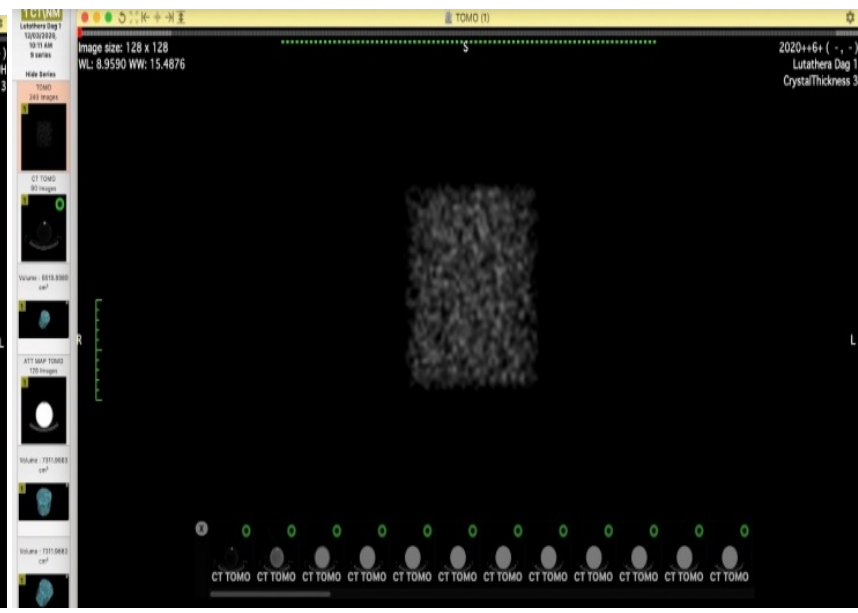

(d)

Figure 13. Scans of phantom at day 0, 6, 13 and 23 respectively. 


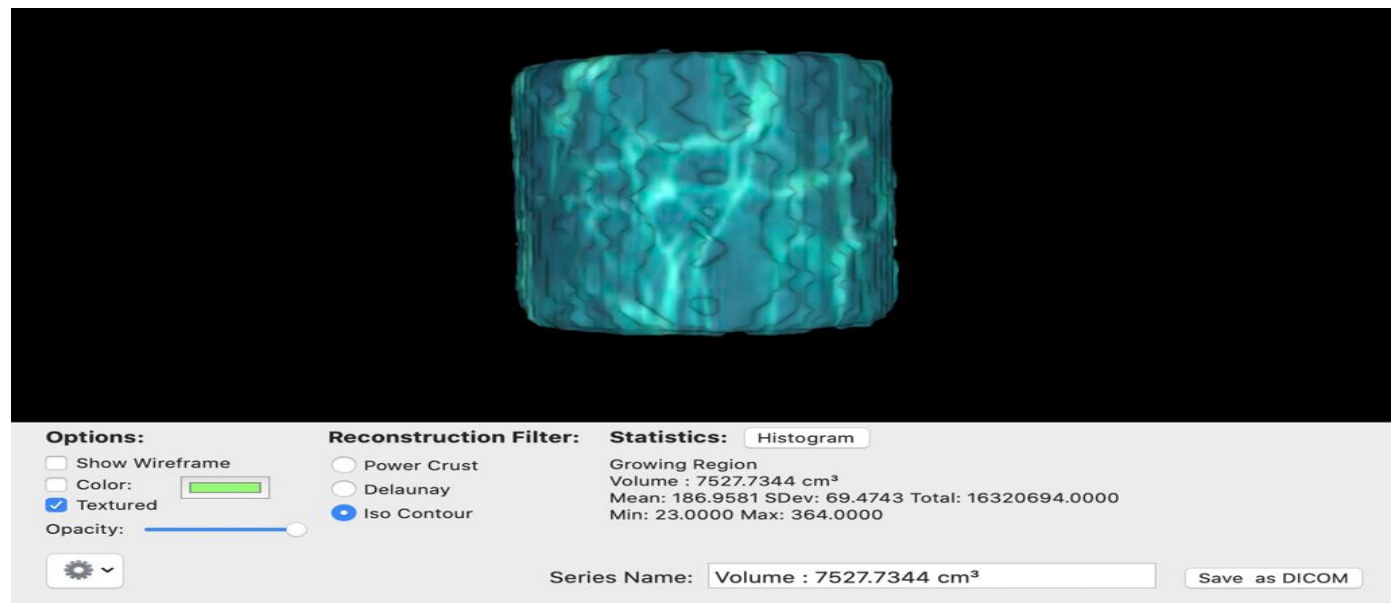

(a)

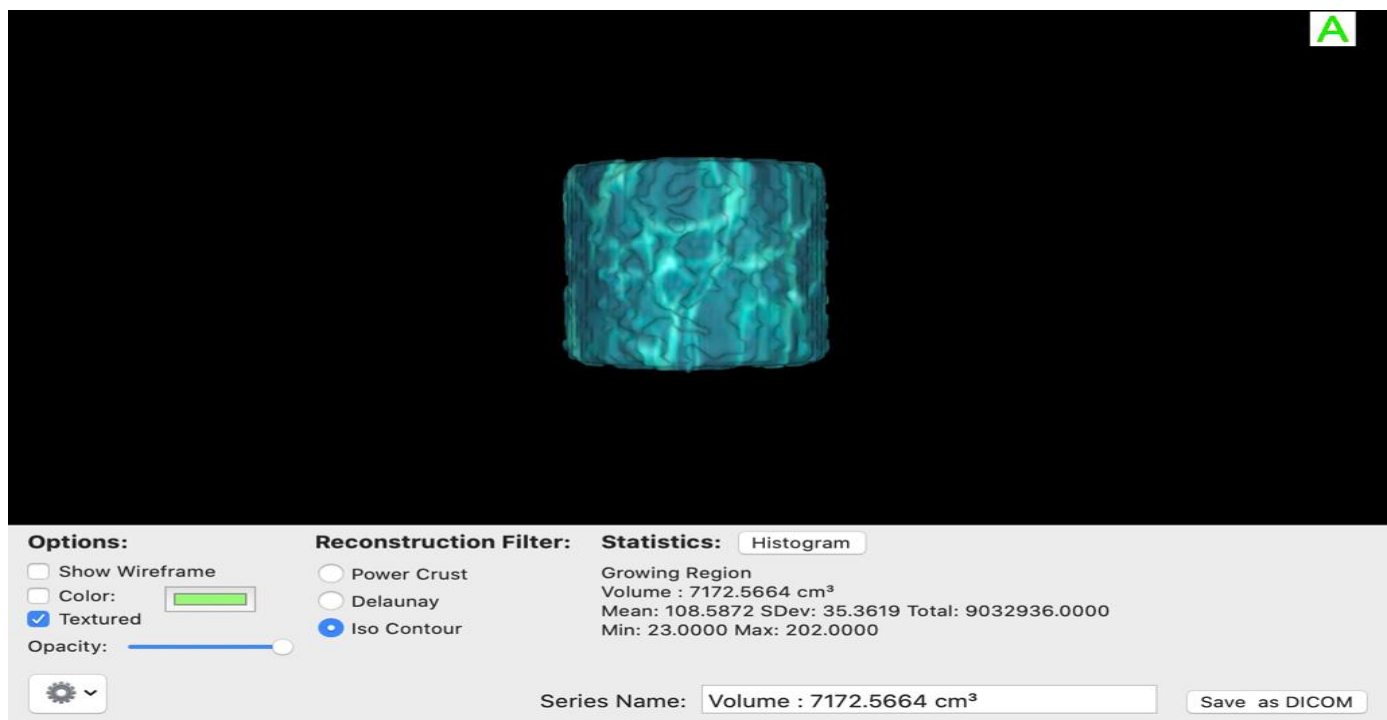

(b)

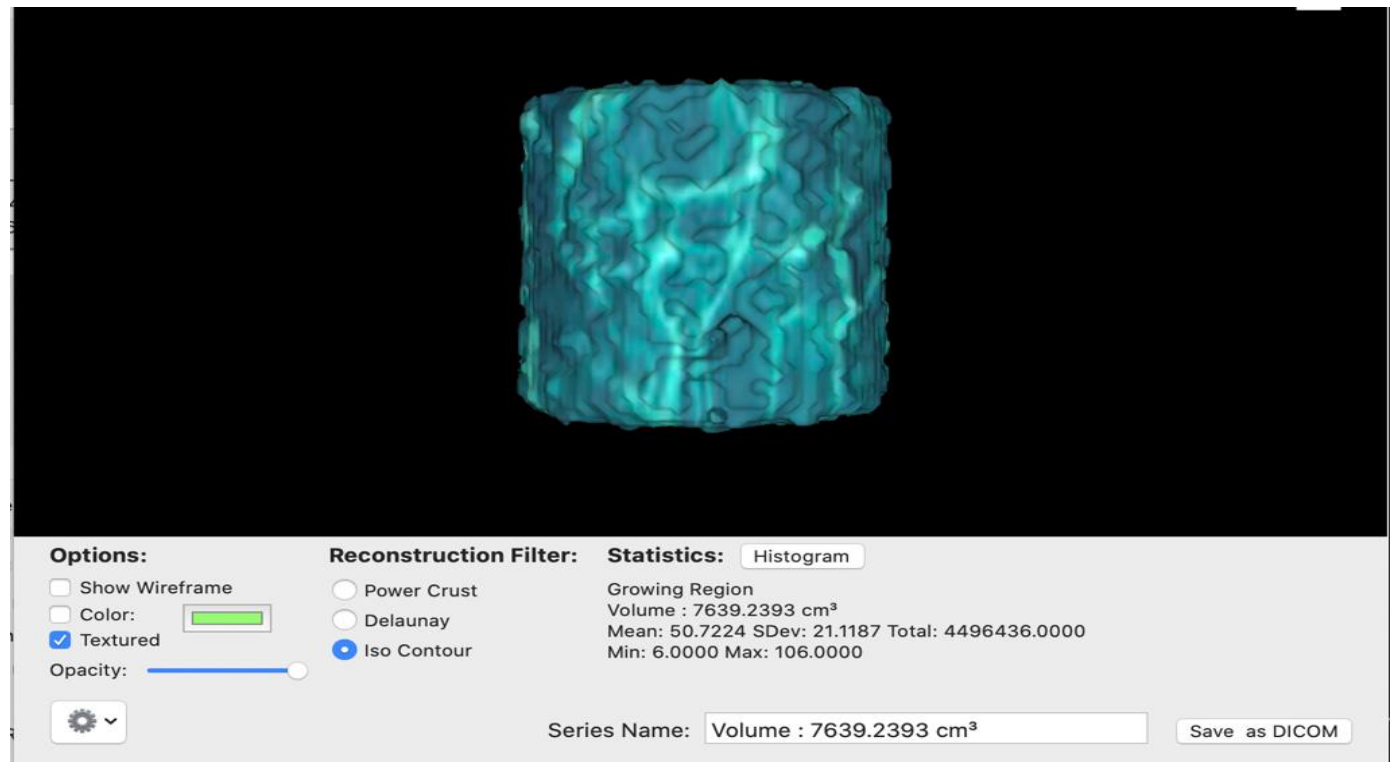

(c) 


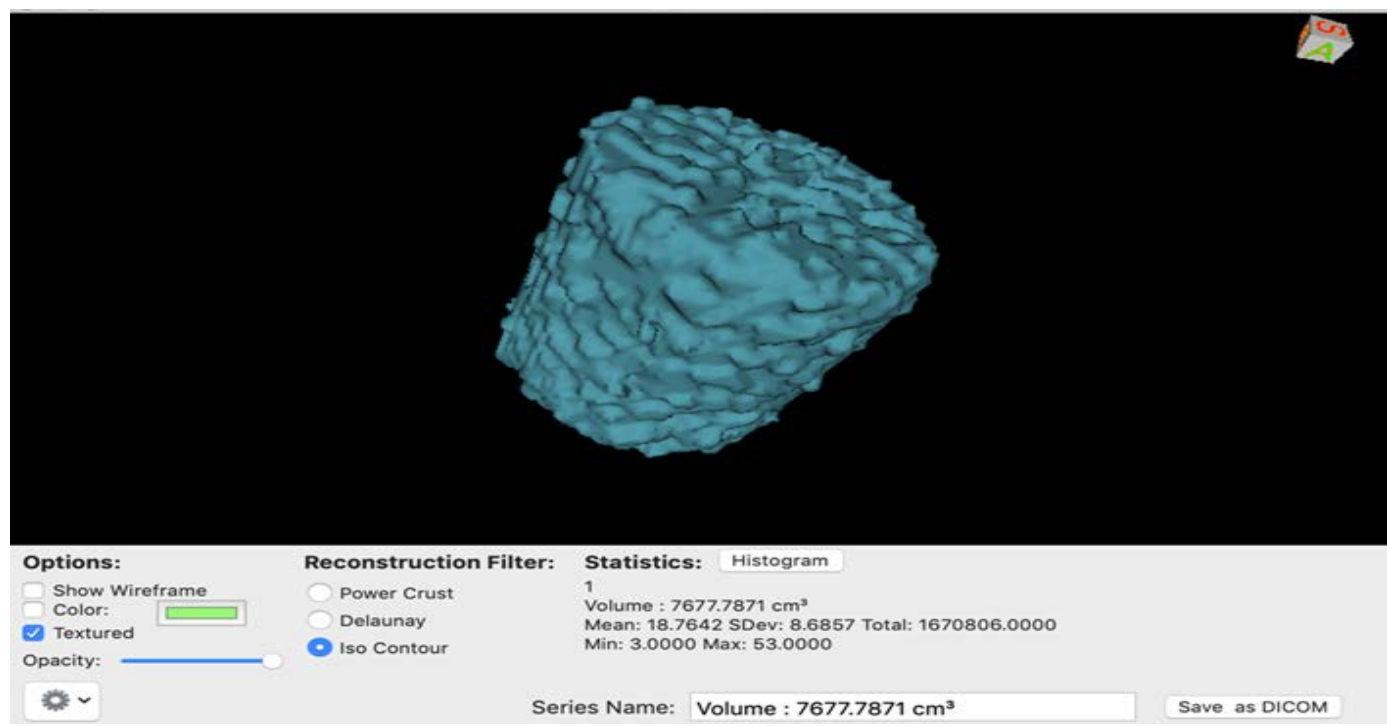

(d)

Figure 14. 3D images acquired from the scanned phantom at day $0,6,13$ and 23 respectively.

Table 8. A table showing the activity at various scan times, measured counts and the calibration factor.

\begin{tabular}{cccc}
\hline Scan date & Activity [MBq] & Measured counts & 3D sensitivity (calibration factor) [cps/MBq] \\
\hline Feb. 18 & 989.19 & $16,320,694$ & 4.62 \\
Feb. 24 & 540.31 & $9,032,936$ & 4.71 \\
Mar. 2 & 261.06 & $4,496,436$ & 4.78 \\
Mar. 12 & 90.75 & $1,670,806$ & 5.29 \\
\hline
\end{tabular}
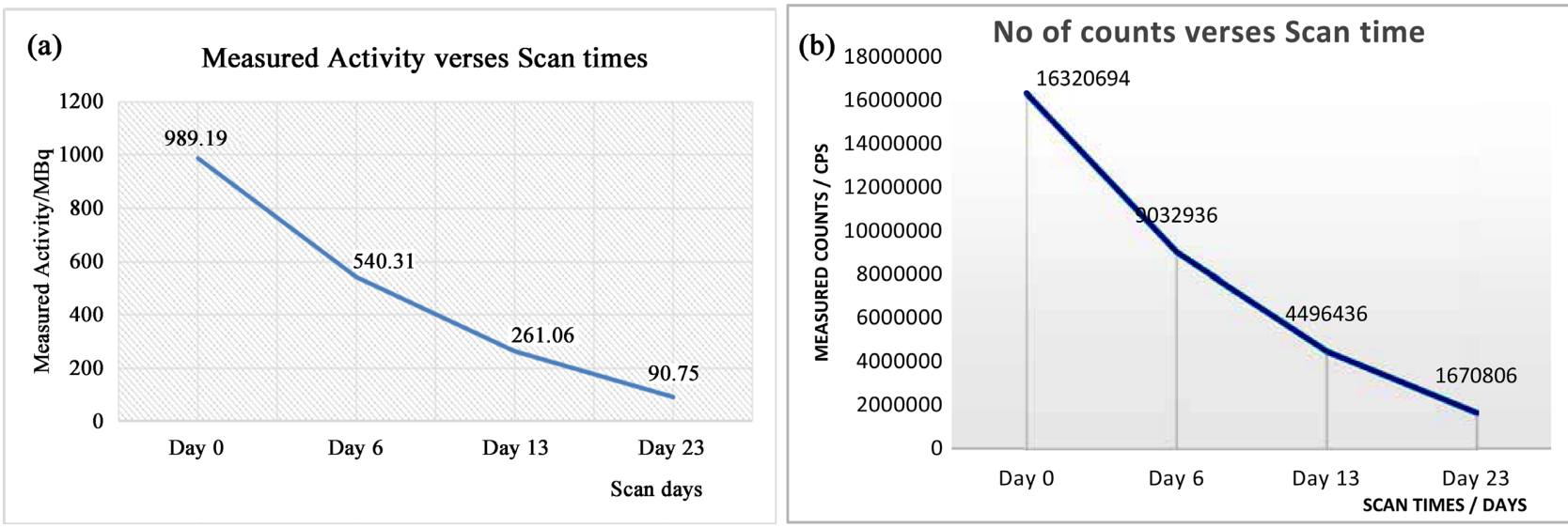

Figure 15. (a) A graph showing measured activity verses scan time; (b) A graph showing number of counts verses scan time.

$3 \mathrm{D}$ images acquired with the total counts obtained from the scanned phantom at days $0,6,13$ and 23 respectively. Table 8 shows the activities at various scan times, measured counts and the calibration factor. Graphs showing measured activity verses scan time and a number of counts verses scan time are shown in Figure 15(a) and Figure 15(b) respectively. The 3D sensitivity verses scan time is also shown in Figure 16. 


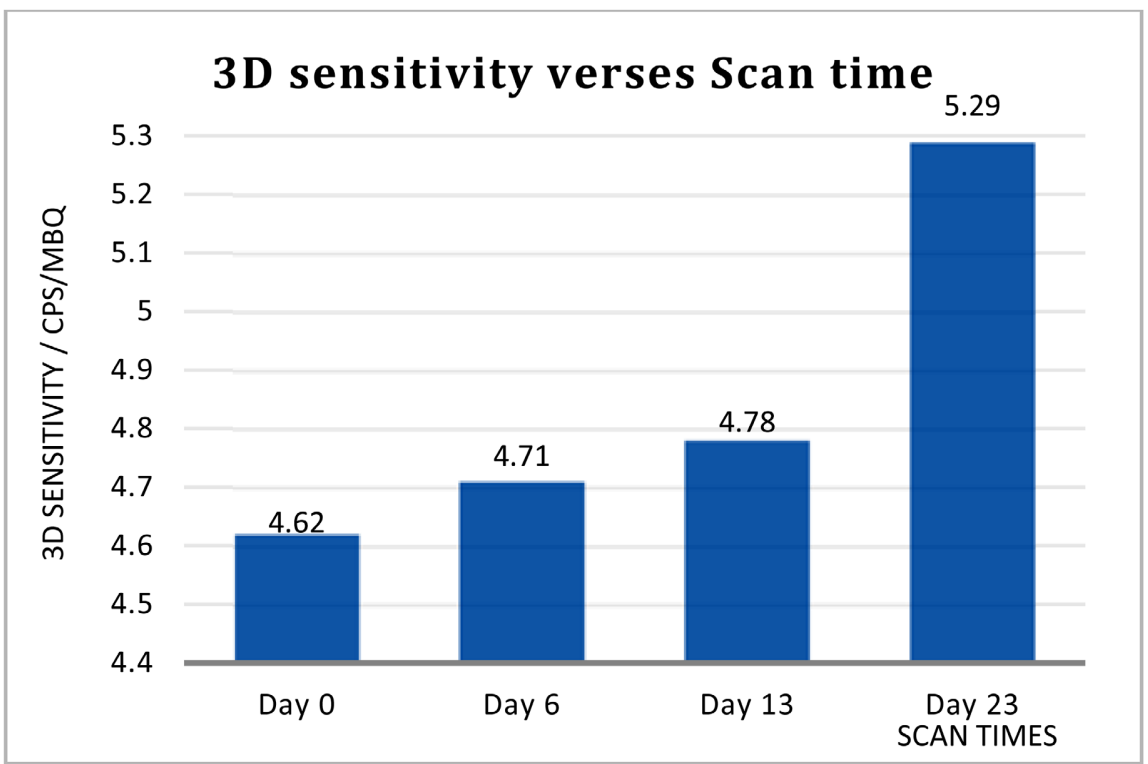

Figure 16. A graph showing the 3D sensitivity verses scan time.

\section{Conclusion}

Several tests were carried out to quality assure the Infinia Hawkeye SPECT/CT and to prepare a workflow for the whole-body dosimetry with Lu-77. The uniformity, energy peak, 3D calibration, 2D sensitivity and CT to NM registration tests were selected to be relevant for the accurate quantification of the system at the St. Olav's hospital. The uniformity for detector 1 was within specifications but that for detector 2 failed. It was approved after iteration calibration of the PM-tubes. The mean 2D sensitivity factor was $5.56 \mathrm{cps} / \mathrm{MBq}$ which corresponds to $1888 \mathrm{c} / \mathrm{MBq}$ for clinical protocol with the measurement time of $340 \mathrm{~s} /$ pixel $(7$ $\mathrm{cm} / \mathrm{min}$ ). The X-ray to SPECT registration was accurate and within specifications. The precision and accuracy of activity quantification utilizing dedicated quality assurance were determined to be adequate for use in dosimetry in clinical practice. Other centers could use the proposed technology to get repeatable ${ }^{177} \mathrm{Lu}$-based treatment dosimetry.

\section{Conflicts of Interest}

The authors declare no conflicts of interest regarding the publication of this paper.

\section{References}

[1] Mattsson, S. and Söderberg, M. (2011) Radiation Dose Management in CT, SPECT/CT and PET/CT Techniques. Radiation Protection Dosimetry, 147, 13-21. https://doi.org/10.1093/rpd/ncr261

[2] Mhiri, A., Slim, I., Ghezaiel, M., Meddeb, I., El Bez, I., Yeddes, I., et al. (2012) L'apport de l'imagerie hybride TEMP/TDM dans la prise en charge du cancer différencié de la thyroïde. Médecine Nucléaire. Imagerie Fonctionnelle et Métabolique, 36, 554-560. https://doi.org/10.1016/j.mednuc.2012.08.008 
[3] Larkin, A.M., Serulle, Y., Wagner, S., Noz, M.E. and Friedman, K. (2011) Quantifying the Increase in Radiation Exposure Associated with SPECT/CT Compared to SPECT Alone for Routine Nuclear Medicine Examinations. International Journal of Molecular Imaging, 2011, Article ID: 897202. https://doi.org/10.1155/2011/897202

[4] Wall, B.F. and Hart, D. (1997) Revised Radiation Doses for Typical X-Ray Examinations: Reports on Recent Review of Doses to Patients from Medical X-ray Examinations in the UK by NRPB. The British Journal of Radiology, 70, 437-439. https://doi.org/10.1259/bjr.70.833.9227222

[5] International Commission on Radiological Protection (ICRP) (1991) 1990 Recommendations of the International Commission on Radiological Protection. ICRP publication No. 60, Pergamon Press, New York.

[6] International Commission on Radiological Protection (ICRP) (2007) 2007 Recommendations of the International Commission on Radiological Protection. ICRP Publication No. 103. Annals of the ICRP, 37, 1-332.

[7] Snyder, W.S., Ford, M.R., Warner, G.G. and Watson, S.B. (1975) "S" Absorbed Dose per Unit Cumulated Activity for Selected Radionuclides and Organs (Part 1). MIRD Pamphlet 11. The Society of Nuclear Medicine, New York.

[8] Sandstrom, M., Garske, U., Granberg, D., Sundin, A. and Lundqvist, H. (2010) Individualized Dosimetry in Patients Undergoing Therapy with ${ }^{177} \mathrm{Lu}$-DOTA-D-Phe ${ }^{1}$ $\mathrm{Tyr}^{3}$-Octreotate. European Journal of Nuclear Medicine and Molecular Imaging, 37, 212-225. https://doi.org/10.1007/s00259-009-1216-8

[9] Garkavij, M., Nickel, M., Sjogreen-Gleisner, K., Ljungberg, M., Ohlsson, T., Wingardh, K., et al. (2010) ${ }^{177} \mathrm{Lu}$-[DOTA0, Tyr3] Octreotate Therapy in Patients with Disseminated Neuroendocrine Tumors: Analysis of Dosimetry with Impact on Future Therapeutic Strategy. Cancer, 116, 1084-1092. https://doi.org/10.1002/cncr.24796

[10] Sward, C., Bernhardt, P., Ahlman, H., Wangberg, B., Forssell-Aronsson, E., Larsson, M., et al. (2010) $\left[{ }^{177} \mathrm{Lu}-\mathrm{DOTA}{ }^{0}-\mathrm{Tyr}^{3}\right]$-Octreotate Treatment in Patients with Disseminated Gastroenteropancreatic Neuroendocrine Tumors: The Value of Measuring Absorbed Dose to the Kidney. World Journal of Surgery, 34, 1368-1372.

https://doi.org/10.1007/s00268-009-0387-6

[11] Fjalling, M., Andersson, P., Forssell-Aronsson, E., Gretarsdottir, J., Johansson, V., Tisell, L.E., et al. (1996) Systemic Radionuclide Therapy Using Indium-111-DTPA-DT Phe1Octreotide in Midgut Carcinoid Syndrome. Journal of Nuclear Medicine, 37, 1519 1521.

[12] Gabriel, M., Andergassen, U., Putzer, D., Kroiss, A., Waitz, D., Von Guggenberg, E., et al. (2010) Individualized Peptide-Related-Radionuclide-Therapy Concept Using Different Radiolabelled Somatostatin Analogs in Advanced Cancer Patients. The Quarterly Journal of Nuclear Medicine and Molecular Imaging, 54, 92-99.

[13] Kwekkeboom, D.J., de Herder, W.W., van Eijck, C.H., Kam, B.L., van Essen, M., Teunissen, J.J., et al. (2010) Peptide Receptor Radionuclide Therapy in Patients with Gastroenteropancreatic Neuroendocrine Tumors. Seminars in Nuclear Medicine, 40, 78-88. https://doi.org/10.1053/j.semnuclmed.2009.10.004

[14] Kwekkeboom, D.J., Kam, B.L., van Essen, M., Teunissen, J.J., van Eijck, C.H., Valkema, R., et al. (2010) Somatostatin-Receptor-Based Imaging and Therapy of Gastroenteropancreatic Neuroendocrine Tumors. Endocrine-Related Cancer, 17, R53-R73. https://doi.org/10.1677/ERC-09-0078

[15] Seregni, E., Maccauro, M., Coliva, A., Castellani, M.R., Bajetta, E., Aliberti, G., et al. (2010) Treatment with Tandem [(90)Y]DOTA-TATE and [(177)Lu] DOTA-TATE of Neuroendocrine Tumors Refractory to Conventional Therapy: Preliminary Results. The Quarterly Journal of Nuclear Medicine and Molecular Imaging, 54, 84-91. 
[16] van Essen, M., Krenning, E.P., Kam, B.L., de Herder, W.W., Feelders, R.A. and Kwekkeboom, D.J. (2010) Salvage Therapy with (177)Lu-Octreotate in Patients with Bronchial and Gastroenteropancreatic Neuroendocrine Tumors. Journal of Nuclear Medicine, 51, 383-390. https://doi.org/10.2967/jnumed.109.068957

[17] Esser, J.P., Krenning, E.P., Teunissen, J.J., Kooij, P.P., van Gameren, A.L., Bakker, W.H., et al. (2006) Comparison of $\left[{ }^{177} \mathrm{Lu}-\mathrm{DOTA}^{0}, \mathrm{Tyr}^{3}\right]$ Octreotate and $\left.{ }^{177} \mathrm{Lu}-\mathrm{DOTA}{ }^{0}, \mathrm{Tyr}^{3}\right]$ Octreotide: Which Peptide Is Preferable for PRRT? European Journal of Nuclear Medicine and Molecular Imaging, 33, 1346-1351.

https://doi.org/10.1007/s00259-006-0172-9

[18] Tolmachev, V., Bernhardt, P., Forssell-Aronsson, E. and Lundqvist, H. (2000) ${ }^{114 \mathrm{~m}} \mathrm{In}$, a Candidate for Radionuclide Therapy: Low-Energy Cyclotron Production and Labeling of DTPA-D-Phe-Octreotide. Nuclear Medicine and Biology, 27, 183-188. https://doi.org/10.1016/S0969-8051(99)00096-7

[19] Rossin, R., Pan, D., Qi, K., Turner, J.L., Sun, X., Wooley, K.L., et al. (2005) ${ }^{64}$ Cu-Labeled Folate-Conjugated Shell Cross-Linked Nanoparticles for Tumor Imaging and Radiotherapy: Synthesis, Radiolabeling, and Biologic Evaluation. Journal of Nuclear Medicine, 46, 1210-1218.

[20] Smith, S.V. (2004) Molecular Imaging with Copper-64. Journal of Inorganic Biochemistry, 98, 1874-1901. https://doi.org/10.1016/j.jinorgbio.2004.06.009

[21] Mansi, L. and Virgolini, I. (2011) Diagnosis and Therapy Are Walking Together on Radiopeptides' Avenue. European Journal of Nuclear Medicine and Molecular Imaging, 38, 605-612. https://doi.org/10.1007/s00259-011-1762-8 